\title{
Heterogeneous Neurological Disorders Associated with the SARS-CoV-2 Infection: A Case Report
}

\author{
Vyacheslav A. Kutashov, PhD, ScD; Olga V. Ulyanova, PhD; Igor S. Protasov, PhD*; \\ Oleg V. Zolotaryov, PhD; Elena S. Ananyeva, PhD; Anna A. Dudina, PhD; \\ Margarita V. Uvarova, $\mathrm{PhD}$ \\ Voronezh State Medical University named after N.N. Burdenko \\ Voronezh, the Russian Federation
}

\begin{abstract}
The novel coronavirus SARS-CoV-2 and the disease it causes, COVID-19, along with damage to the respiratory system, can lead to disorders of the central and peripheral nervous system, as well as the muscular system. The article presents literature data and the authors' observations of the course of neurological disorders in a patient with COVID-19. This study found that there is a link between the severity of COVID-19 and the intensity and frequency of neurological disorders.(International Journal of Biomedicine. 2021;11(1):61-64.)
\end{abstract}

Key Words: SARS-CoV-2 $\bullet$ pandemic $・$ status epilepticus $\bullet$ viral pneumonia $\bullet$ myasthenic syndrome

For citation: Kutashov VA, Ulyanova OV, Protasov IS, Zolotaryov OV, Ananyeva ES, Dudina AA, Uvarova MV. Heterogeneous Neurological Disorders Associated with the SARS-CoV-2 Infection: A Case Report. International Journal of Biomedicine. 2021;11(1):61-64. doi:10.21103/Article11(1)_CR2

\section{Abbreviations}

ARDS, acute respiratory distress syndrome; HLA, the human leukocyte antigen; IL, interleukin; IgM, immunoglobulin M; CPK, creatine phosphokinase; LDH, lactate dehydrogenase; ACE2, angiotensin converting enzyme 2; CT, computed tomography; CTA, computed tomography angiography; MRA, magnetic resonance imaging; ENMG, electroneuromyography.

\section{Introduction}

The well-functioning health systems of the developed world have been deeply shaken by the year 2019. ${ }^{(1-3)}$ COVID-19 cases have been reported everywhere, and on March 11, 2020, the World Health Organization declared COVID-19 as a global pandemic. The clinical picture of the novel coronavirus SARS-CoV-2 is characterized primarily by disorders of the respiratory system (in $91.1 \%$ of the cases, the development of severe ARDS is observed as a leading complication), febrile syndrome ( $83 \%-99 \%$ of the cases), and pathology of the skin

*Corresponding author: Igor S. Protasov, PhD. Voronezh State Medical University named after N.N. Burdenko. Voronezh, Russia.E-mail:protasovis@mail.ru integument, gastrointestinal tract (up to $15 \%$ of the cases), urinary and nervous systems. ${ }^{(4)}$

Based on the available scientific data, the concept of a multisystem response of the body to the penetration of the SARS-CoV-2 virus has been developed. We have tried to summarize the known pathogenetic features of SARS-CoV-2 virulence. ${ }^{(5-8)}$ As currently established, SARS-CoV-2 is tropic to type II alveolocytes and enterocytes of the small intestine. The virus enters the cell due to the attachment of the peplomer S-protein. Once in the cell, the virus is preactivated by serine proteases (furin and TMPRSS2); then the active phase of the viral RNA replication begins with several independent parts. After the completion of the replication phase, the vesicles containing the virion fuse with the plasmatic membrane of the cell, and the virus is released. At the moment serine 
proteases are activated, cytokines and IL-2 are released, triggering the activation of CD4 lymphocytes, which in turn activate Th1 lymphocytes. However, due to the mimicry of the virus, the immune system cannot adequately recognize the amount of viral antigens, probably, among other things, due to the activation of immune control and the launch of Thsuppressors, which may be one of the causes of leukopenia. This hypothesis explains the high contagiousness of the virus during the latent period of the disease. After the virus is isolated from the "primary" cell of the type II alveocyte, it is carried out with the blood flow, and enterocytes of the small intestine become the second most important target. The mechanism of penetration into enterocytes is similar. The local response activates B-lymphocytes and triggers Th2-lymphocytes, promoting the release of IL-4 and IL-5 into the blood. The humoral response (3-8 days) begins to form; IgM is released. In response, the complement system is activated; the forming, circulating immune complexes are captured by mast cells, increasing the release of histamine and serotonin into the blood, thereby triggering a systemic inflammatory response. After a few days of the latent period, changes in metabolic processes begin in the target cells; the normal rhythm of the cell's work is disrupted, including the synthesis of the components necessary for functioning, and the process of apoptosis starts. The alveolar macrophages are activated with the release of proinflammatory agents IL-6, IL-8, and TNF-alpha. Chemoattractants are released into the blood, stimulating the movement of monocytes and neutrophils from the blood through the endothelium and alveolar epithelium, which finally causes a violation of ventilation and perfusion processes with the accumulation of fluid in the alveoli. In turn, the leukocytes themselves are a source of leukotrienes, proteases, and platelet aggregation factor, which causes alveolar fibrin deposition, the formation of hyaline membranes, and the formation of microthrombus in the vascular bed of the lungs, forming toxic edema and acute respiratory distress syndrome. Further progression of respiratory failure is associated with the addition of bacterial flora. Thus, based on the currently available research data, it can be assumed that the rapid development, multisystem damage, rapid increase in the systemic inflammatory response syndrome, and cytokine storm are caused by the compromised states of the immune system. Since a cascade mechanism is launched, which is lightning-fast in nature, the immune system lacks time to react in a timely and adequate manner. The probable reasons for this are the following: misidentification of the virus due to mimicry of the SARSCov-2 S-protein to ACE2 and its presentation as an antigen by antigen-presenting cells; rapid virus replication in type II alveocytes (primary gateway for infection) with subsequent blockage of regional lymph nodes due to the peculiarities of the formation of the immune response, which contributes to an increase in local blood supply and the influx of lymphocytic cells from the recirculating pool with "inhibition" of cellular immunity; rapid migration and subsequent dissemination of replicated SARS-CoV-2 with blood flow into the small intestine; and "secondary" virus replication in enterocytes with "inhibition" of humoral immunity. Since the HLA system is responsible for the quality of antigen-presenting cells, it is logical to assume that the intensity and massiveness of the immune system response is genetically determined, especially in patients with autoimmune diseases. Thus, as a screening for the activation of the immune response (or its clearly high level in the presence of an autoimmune disease) and a high risk of developing severe forms of COVID-19 in the future, it is possible to use the levels of the so-called non-classical HLA complexes: HLA-MICA as an early signal of the immune response to the infectious damage; HLA-E as an attempt by the body to cope with a cascade of proliferative immune responses; HLA-B27 as an indicator of the number and activity of antigen-presenting cells. Taking into account the population characteristics of HLA, it is natural to assume the peculiarities of the immune response in individuals with different blood groups. Thus, it is likely that individuals with hemagglutinins (blood groups A (II), B (III), and AB (IV)) may have more severe forms of COVID-19 due to mimicry of the S-protein of SARS-CoV-2, but this assumption is hypothetical and requires further detailed study. However, it is clear that the presence of chronic diseases with a constant level of stimulation of the immune system contributes to hyperresponsiveness, the increased release of cytokines, and the development of severe forms of novel coronavirus infection (this is evidenced by the positive effect of the use of glucocorticosteroids). Given the features of SARS-CoV-2 replication, it is also likely to suggest in the near future the following options of treatment-blockage of virus replication: blockage of CD147 with monoclonal antibodies (mepolizumab) in the absence of signs of bacterial infection; blockage of serine proteases (furin, TMPRSS2) with Kalistat; blockage of RNA replication (Avifavir). The assumptions made are hypothetical and, of course, require further research. ${ }^{(4)}$

It is noteworthy that a number of viral infections can be accompanied by direct damage to the skeletal muscles. On the other hand, skeletal muscle damage can be secondary in severe viral infection, especially complicated by sepsis, multiple organ failure, and acute respiratory distress syndrome. L. Mao et al., ${ }^{(9)}$ on the basis of an increase in the levels of CPK and $\mathrm{LDH}$, diagnosed the involvement of the muscular system in $10.7 \%$ of patients with a predominance of the severe form of the disease (19.3\% and 4.8\%). In groups with mild and severe cases of COVID-19, the levels of CPK and LDH were reliably higher in patients with muscle weakness than without it. At the same time, in patients with muscle symptoms, the level of CPK and LDH was significantly higher in the severe form of the disease than in the group with a mild form. This distribution suggests that the cause of damage to the muscular system could be not only a direct viral effect, but also a general severe condition with metabolic disorders. An increase in laboratory markers reflecting skeletal muscle and myocardial injury in COVID-19 has been noted by Q. Ruan et al. ${ }^{(10)}$ They, as well as L. Mao et al., ${ }^{(9)}$ drew attention to the relationship between the severity of the condition and increased myoglobin content. The mechanisms of skeletal muscle damage in COVID-19 are not entirely clear. Presumably, they can be associated with ACE2 receptors, which are widely represented in skeletal muscle and myocardium; their expression increases during 
viral infection and the severe condition with increased muscle tissue decay. Along with this, G. Baird and T. Montine consider the excessive production of cytokines during inflammation as a direct damaging factor of muscle tissue. ${ }^{(11)}$ The role of a pathological autoimmune reaction in polyclonal stimulation of the immune system by a virus with cross-damage of skeletal muscle antigens is not excluded.(12)

Neurological disorders can be divided into 3 types:

1) Manifestations of the central nervous system (dizziness, headache, impaired consciousness, acute cerebrovascular event, ataxia, and convulsions)

2) Manifestations of the peripheral nervous system (taste, smell, and vision disorders; neuralgias)

3) Damage to the musculoskeletal system ${ }^{(13)}$

During the COVID-19 pandemic, hospitalized patients with neurological symptoms should be examined for COVID-19 to make a quick and correct diagnosis. In addition, it is essential to preserve as much as possible the quality of medical care for patients with neurological diseases, especially with acute damage to both the central and peripheral nervous systems, while taking all precautions to avoid transmission of a novel coronavirus infection to the medical personnel and patients. ${ }^{(14)}$

\section{Case Presentation}

\section{Patient Information}

A 24-year-old man, on May 19, 2020, presented by ambulance to the intensive care unit of the Voronezh City Clinical Emergency Hospital No.1 with a diagnosis of "seizures of unknown genesis." Complaints: double vision, unsteadiness when walking, numbness of the lower limbs, and discomfort while swallowing.

\section{Clinical Findings and Diagnostic Assessment}

Status epilepticus. The patient was transferred to artificial lung ventilation (ALV) - mechanical ventilation, CT scan of the lungs showed a pattern characteristic of viral pneumonia caused by the novel coronavirus infection COVID-19. A COVID-19 coronavirus RNA test confirmed the diagnosis. CTA: a picture of complete trifurcation of the right internal carotid artery. The asymmetry of the vertebral arteries $\mathrm{D}>\mathrm{S}$. CT scan of the brain did not reveal any pathology. MRI of the brain revealed no pathological changes. Lumbar puncture: general analysis of cerebrospinal fluid (CSF), biochemical analysis, and polymerase chain reaction results were without pathology. CSF seeding showed no pathology either. ALV was performed for 5 days.

Clinical Diagnosis: "Status epilepticus as of May 19, 2020. Confirmed COVID-19: severe disease course, bilateral viral pneumonia."

\section{Treatment}

Convulex, Encorate, Sulbactam, Azithromycin, Hydroxychloroquine, Enoxaparin, Sterofundin, Omeprazole, and Tocilizumab.

\section{Outcome and Follow-up}

Diagnosis at discharge: "Confirmed COVID-19: severe disease course, bilateral viral pneumonia regressed, status epilepticus as of May 19, 2020.”
The patient stayed at home for two days; after that, he experienced pronounced double vision, numbness of the lower limbs, dizziness, and unsteadiness when walking. The patient was readmitted to the neurological department for patients with violation of cerebral circulation at the Voronezh City Clinical Emergency Hospital No.1

\section{Clinical Findings and Diagnostic Assessment}

The patient's previous history also included bronchial asthma and atopic dermatitis.

Objectively: A moderate-severe condition. Skin: rash in the area of the anterior abdominal wall and shoulder joints. Heart sounds are sonorous, rhythmic. Blood pressure - 140/80 $\mathrm{mmHg}$, heart rate $-76 \mathrm{bpm}$. In the lungs, breathing is vesicular. Respiratory rate -17 per minute. The abdomen is soft, free of pain. The liver and spleen are not palpable. The stool and diuresis are without pathology. The body temperature $-36.6^{\circ} \mathrm{C}$.

Neurological status: Wakefulness level - clear consciousness; there is an orientation to self, location, and time. Speech is preserved. There are no meningeal signs. Pupils $\mathrm{D}=\mathrm{S}$; direct and concomitant reaction to light is preserved. Eye slits $\mathrm{D}>\mathrm{S}$; congenital left-sided hemi-ptosis; full eyeball movement; horizontal nystagmus; diplopia when looking in all directions. The nasolabial folds are symmetrical. The tongue is on the midline. Hearing is preserved. Swallowing and phonation are not disturbed. Active pharyngeal reflexes $\mathrm{D}=\mathrm{S}$; deep reflexes $\mathrm{D}=\mathrm{S}$. No pareses. Muscle tone is not changed. There are no pathological signs. The patient performs coordination tests with misses on both sides. In the Romberg posture, swaying occurs. Sweating is normal. Dermographism is pink. There is knee-high hypoesthesia of the lower limbs.

The additional examination (brain MRI, duplex ultrasound of the brachiocephalic artery, EEG during one hour, ENMG) without pathology.

\section{Treatment}

Pulse therapy with glucocorticosteroids, anticholinesterase drugs, neuroprotection, and metabolic therapy. After the treatment, there was no positive dynamics; double vision did not decrease, and left-sided hemianopsia also began to appear.

Examination of the fundus revealed no pathology. Needle ENMG was repeated. The blink reflex showed an inconsistent increase in R2 component latency with stimulation on the left. The jitter from the circular muscle of the eye on the left revealed an irregular increase in the interpeak intervals without dropout of second potentials.

The ENMG picture allows us to discuss the involvement in the pathological process of the ipsilateral spinal tract of the $\mathrm{V}$ nerve/intercalary neurons reticular formation, mainly on the left in the brain stem. Myasthenic-type neuromuscular transmission disorder in the circular muscle of the eye was not detected; however, there were dropout units with transmission disorder. Given the data obtained, we decided to conduct 5 plasmapheresis sessions. The double vision was gone. At discharge, the patient's condition was completely stabilized with no pathology in the neurological status.

Clinical Diagnosis: 'Encephalopolyneuropathy associated with the consequences of COVID-19; initial manifestations of myasthenic syndrome (according to ENMG data); status epilepticus as of May 19, 2020.” 
Thus, the damage to the nervous system in the patient appeared as a result of the COVID-19 infection and was of a heterogeneous nature.

\section{Discussion}

Over the past few months, our understanding of the transmissivity, pathogenesis, and clinical heterogeneity of coronavirus pneumonia caused by SARS-CoV-2 has been significantly expanded and modified. The few publications devoted to the development of neurological conditions associated with COVID-19 (meningoencephalitis, acute cerebrovascular pathology, Guillain-Barré syndrome, etc.) do not give an exhaustive answer, whether those conditions are a consequence of the direct neurotropic action of the virus or are mediated by an immune response and other reactions (including pro-inflammatory and pro-thrombotic influences). Besides, like other infectious diseases, SARS-CoV-2 can lead to the clinical realization of asymptomatic comorbidities (mostly cardiovascular and endocrine disorders) and their aggravation, with a risk of a fatal outcome.

The COVID-19 pandemic poses several tasks for the neuroscience community: 1) to provide adequate therapy for patients with diseases of the nervous system (cerebrovascular diseases) ${ }^{(15)}$ various forms of dementia, Parkinson's disease, amyotrophic lateral sclerosis) who are at high risk of developing complications; 2) to formulate algorithms for timely diagnostic and therapeutic care for patients with acute neurological conditions in the context of COVID-19 pandemic (acute cerebrovascular accident, exacerbation of multiple sclerosis, myasthenic crisis, status epilepticus); 3) to ensure the prevention of complications caused by coronavirus infection among patients receiving immunosuppressive therapy for various autoimmune diseases, as well as cancerous lesions of the nervous system. Despite the increasing number of publications on this topic, to date, the information on the neurological aspects of COVID-19 is incomplete and further research is required.

\section{Competing Interests} interests.

The authors declare that they have no competing

\section{References}

1. WHO. Coronavirus Disease (COVID-19). Situation Report - 209. Available at https://www.who.int/docs/defaultsource/coronaviruse/situation-reports/20200816-covid-19sitrep-209.pdf?sfvrsn=5dde1ca2_2. Data as received by WHO from national authorities by 10:00 CEST, 16 August 2020

2. WHO. Coronavirus disease (COVID-19). Available at https://www.who.int/docs/default-source/coronaviruse/ situation-reports/20200921-weekly-epi-update-6. pdf?sfvrsn=d9cf9496_4. Data as received by WHO from national authorities, as of 10 am CEST 20 September 2020
3. Cutler DM, Summers LH. The COVID-19 Pandemic and the \$16 Trillion Virus. JAMA. 2020 Oct 20;324(15):14951496. doi: 10.1001/jama.2020.19759.

4. Karpova OV, Udalov YuD, Samoylov AS, Kudryavtsev RA. [Features of Case Management with Neuromuscular Disease During COVID-19. Clinical Impression]. Journal of Clinical Practice. 2020;11(2):107-117. doi: 10.17816/ clinpract34580. [Article in Russian].

5. Rao GHR. SARS-CoV-2 Biochemistry, Transmission, Clinical Manifestations, and Prevention. International Journal of Biomedicine. 2020; 10(4):303-311. doi: 10.21103/ Article10(4)_GE

6. Burki T. The origin of SARS-CoV-2. Lancet Infect Dis. 2020 Sep;20(9):1018-1019. doi: 10.1016/S1473-3099(20)30641-1.

7. Wang H, Li X, Li T, Zhang S, Wang L, Wu X, Liu J. The genetic sequence, origin, and diagnosis of SARS-CoV-2. Eur J Clin Microbiol Infect Dis. 2020 Sep;39(9):1629-1635. doi: 10.1007/s10096-020-03899-4.

8. Severe Covid-19 GWAS Group, Ellinghaus D, Degenhardt F, Bujanda L, Buti M, Albillos A, Invernizzi P, et al. Genomewide Association Study of Severe Covid-19 with Respiratory Failure. N Engl J Med. 2020 Oct 15;383(16):15221534. doi: 10.1056/NEJMoa2020283.

9. Mao L, Jin H, Wang M, Hu Y, Chen S, He Q, Chang J, Hong C, Zhou Y, Wang D, Miao X, Li Y, Hu B. Neurologic Manifestations of Hospitalized Patients With Coronavirus Disease 2019 in Wuhan, China. JAMA Neurol. 2020 Jun 1;77(6):683-690. doi: 10.1001/jamaneurol.2020.1127.

10. Ruan Q, Yang K, Wang W, Jiang L, Song J. Clinical predictors of mortality due to COVID-19 based on an analysis of data of 150 patients from Wuhan, China. Intensive Care Med. 2020 May;46(5):846-848. doi: 10.1007/s00134-020-05991-x.

11. Baird GS, Montine TJ. Multiplex immunoassay analysis of cytokines in idiopathic inflammatory myopathy. Arch Pathol Lab Med. 2008 Feb;132(2):232-8. doi: 10.1043/1543-2165(2008)132[232:MIAOCI]2.0.CO;2.

12. Rodionova OV, Sorokoumov VA. Neurological diseases in the context of the COVID-19 pandemic (review of the literature). The Scientific Notes of the Pavlov University. 2020;27(2):18-24. [Article in Russian].

13. Gusev EI, Martynov MY, Boyko AN, Voznyuk IA, Latsh NY, Sivertseva SA, Spirin NN, Shamalov NA. Novaya koronavirusnaya infektsiya (COVID-19) i porazhenie nervnoi sistemy: mekhanizmy nevrologicheskikh rasstroistv, klinicheskie proyavleniya, organizatsiya nevrologicheskoi pomoshchi [Novel coronavirus infection (COVID-19) and nervous system involvement: pathogenesis, clinical manifestations, organization of neurological care]. Zh Nevrol Psikhiatr Im S S Korsakova. 2020;120(6):7-16. doi: 10.17116/ jnevro20201200617. [Article in Russian].

14. Tanashyan MM. Kuznetsova PI, Raskurazhev AA. [Neurological aspects of COVID-19]. Annaly Klinicheskoi i Eksperimental'noi Nevrologii. 2020;14(2):62-69. [Article in Russian].

15. Kutashov VA., Protasov IS. Recommendations for the management of patients with acute cerebrovascular accidents in the context of the COVID-19 pandemic. Voronezh State Medical University named after N.N.Burdenko; 2020. [In Russian]. 